\title{
Review: Image Enhancement and Background Detection using Mathematical Morphology [MM]
}

\author{
Payal T. Gadare ${ }^{1}$, Prof. Nitin Raut ${ }^{2}$ \\ M. Tech Scholar, Guru Nanak Institute of Engineering \& Technology, Nagpur, India ${ }^{1}$ \\ Professor, Guru Nanak Institute of Engineering \& Technology, Nagpur, India ${ }^{2}$
}

\begin{abstract}
This paper deals with analysis of image Enhancement and background detection used in Mathematical Morphological [MM]. In this paper, Low intensity images have been processed through Block Analysis and Morphological Transformation. Basically, Weber's Law Operator is used to analyze Low intensity images which are carried out by two methods such as Image background detection by block analysis while second operator utilize opening by reconstruction to define multi background notion. Basic Morphological Transformations are like Erosion, Dilation, Compound operation such as Opening by reconstruction, closing by Reconstruction and Block Analysis is used to detect the background of images. Analysis of above mention methods illustrated through the processing of images with filtering techniques along with different dark background images.
\end{abstract}

Keywords: Block Analysis, Opening by Reconstruction, Opening operation, Morphological Transformation.

\section{INTRODUCTION}

In this Paper, This approach is used to enhanced the Low Intensity image along with the concept is to detect the background in images Lighting. Various Mathematical Morphology $[\mathrm{MM}]$ is used to enhance digital images with Low Intensity condition.

Mathematical morphology approach is based on set theoretic concepts of shape. In morphology, Objects present in an image are treated as sets. There are standard techniques like histogram equalization histogram stretching for improving the poor contrast of the degraded image. In the First method the background images in Low Intensity of grey level images can identified by the use of morphological operators.

Lately image enhancement has been carried out by the application based on Weber's Law [2]. Later on erosion, dilation, Opening (erosion followed by dilation), closing (dilation followed by erosion) and opening by reconstruction method is followed. In this paper, firstly we give introduction about various morphological operators and then we apply them on a bad light image and extract the background of that image and then improve contrast of that image.[3]

\subsection{Morphology}

Morphology is a technique of image processing based on shapes. The value of each pixel in the output image is based on a comparison of the corresponding pixel in the input image with its neighbors. By choosing the size and shape of the neighbourhood, can construct a morphological operation that is sensitive to specific shapes in the input image.

Mathematical morphology is a set-theoretical approach to multi-dimensional digital signal or image analysis, based on shape. It is a theory and technique for the analysis and processing of geometrical structures, based on set theory, lattice theory, topology, and random functions. It is most commonly applied to digital images, but it can be employed as well on graphs, surface meshes, solids, and many other spatial structures.

It is also the foundation of morphological image processing, which consists of a set of operators that transform images according to the above characterizations. Mathematical morphology was originally developed for binary images, and was later extended to scale functions and images. The subsequent generalization to complete lattices is widely accepted today as MM's theoretical foundation.

\section{PROPOSED WORK}

In this proposed work, background of the Image compute through two approximations using Matlab. The first approximations are block analysis, while the second proposal is morphological operations.

\subsection{Background Detection by Block Analysis:}

In this analysis, first of all we will read an image as input image with Low Intensity condition having dimension ( $\mathrm{N}$ $\mathrm{X} \mathrm{N}$ ) and divide it into several blocks (say ' $\mathrm{n}$ ' block length) and from each block we will determine the background and apply the weber's law and thereby we obtain an enhanced image

For each analyzed block, maximum (Mi) and minimum (mi) values are used to determine the background criteria $\mathrm{Ti}$ in the following way: 
Vol. 6, Issue 1, January 2017

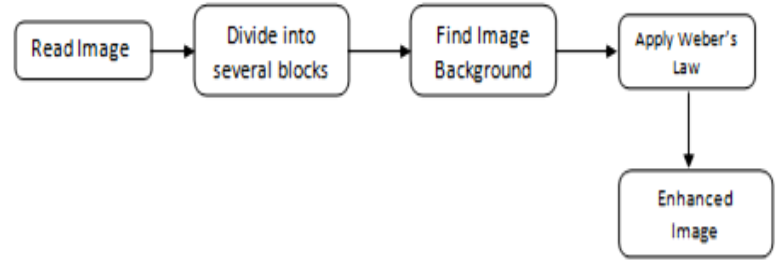

fig. 2.1.1 Block diagram of Background Detection by Block Analysis

\section{Weber's Law:}

Weber's law defines contrast and introduces the concept of Just-Noticeable difference [JND]. Weber's law are more sensitive to light intensity changes in low light levels than in strong ones. The contrast sensitivity is approximately independent of the background luminance. Relative changes in luminance are important. Weber's law tends to break down for very dark and very bright luminance levels[13]. In psycho-visual studies, the contrast $\mathrm{C}$ of an object with luminance Lmax against its surrounding luminance Lmin is defined as follows[2][4].

$$
\mathrm{C}=\frac{\mathrm{L}_{\max }-\mathrm{L}_{\min }}{\mathrm{L}_{\min }}
$$

If $\mathrm{L}=\mathrm{Lmin}$ and $\Delta=\mathrm{L}_{\max }-\mathrm{L}_{\min }$ so it can be written as follows

$$
\mathrm{C}=\frac{\Delta L}{L}
$$

In the above Equation indicates that $\Delta(\log \mathrm{L})$ is proportional to C. Therefore, Weber's law can be expressed as

$$
C=k \log L+b \quad L>0
$$

Where $\mathrm{k}$ and $\mathrm{b}$ are constants, $\mathrm{b}$ being the background. In this case, an approximation to Weber's law is considered by taking the luminance $\mathrm{L}$ as the grey level intensity of a function (image); in this way, above expression is written as follows

$$
C=k \log f+b \quad F>0
$$

2.2 Background detection using morphological operators:

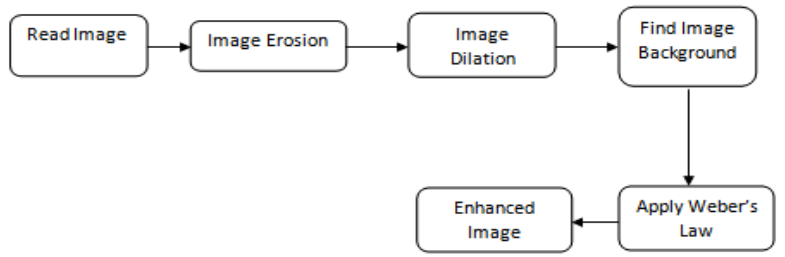

fig. 2.2.1 Block diagram of Background Detection by Erosion \& Dilation

\section{Morphological operations:}

In a morphological operation, the value of each pixel in the output image is based on a comparison of the corresponding pixel in the input image with its neighbors. By choosing the size and shape of the neighborhood, Dilation and erosion are two fundamental morphological operations. Dilation adds pixels to the boundaries of objects in an image, while erosion removes pixels on object boundaries. The number of pixels added or removed from the objects in an image depends on the size and shape of the structuring element used to process the image. Erosion and Dilation can also be interpreted in terms of whether a structuring element (SE) hits or fits an image (region) as follows

For Dilation, The resulting image $\mathrm{g}(\mathrm{x}, \mathrm{y})$, given an input image $f(x, y)$ and a SE will be

$$
g(x, y)=\left\{\begin{array}{l}
1 \text { if se hits f } \\
0 \text { otherwise }
\end{array}\right.
$$

For all $\mathrm{x}$ and $\mathrm{y}$,

For Erosion, The resulting image $\mathrm{g}(\mathrm{x}, \mathrm{y})$, given an input image $f(x, y)$ and a SE will be

$$
g(x, y)=\left\{\begin{array}{l}
1 \text { if se fits } f \\
0 \text { otherwise }
\end{array}\right.
$$

For all $\mathrm{x}$ and $\mathrm{y}$,

\section{Morphological Operation}
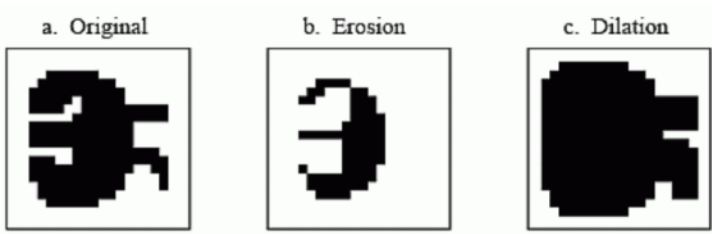

Fig.2.2.2(a)Original Image, Fig (b)Erosion Operation on Image (a).Fig(c) Dilation on image (a).

\section{IMAGE BACKGROUND DETERMINATION USING THE OPENING BY RECONSTRUCTION}

Opening by reconstruction is used to define multi background notion. Opening by reconstruction requires removing pixels of the foreground from an image by specified structuring element. It illustrates through morphological transformations which generate new contours when the structuring element is increased and not necessarily need to apply block analysis and morphological erosion and dilation. When morphological erosion or dilation are used with large sizes of to reveal the background, inappropriate values may be obtained. However, in MM, there is other class of transformations that allows the filtering of the image without generating new components; these transformations are called transformations by reconstruction. In our case, the opening by reconstruction is our choice because touches the regional minima and merges regional maxima. This characteristic allows the modification of the altitude of regional maxima when the size of the structuring element increases. This effect can be used to detect the background criteria $\tau(x)$ i.e.,

$$
\tau(x)=\tilde{y} \mu(f)(X)
$$

When considering the opening by reconstruction to detect the background, one further operation is necessary to detect the local information given by the original function 
Vol. 6, Issue 1, January 2017

The morphological transformation proposed for this task is the erosion size , $\mu=1$ i.e.

$$
b(x)=\varepsilon_{1}\left[\tilde{Y}_{\mu}(f)\right](X)
$$

Given that the morphological erosion tends to generate new information when the structuring element is enlarged, in this study, the image background was computed by using only the morphological erosion size 1 .

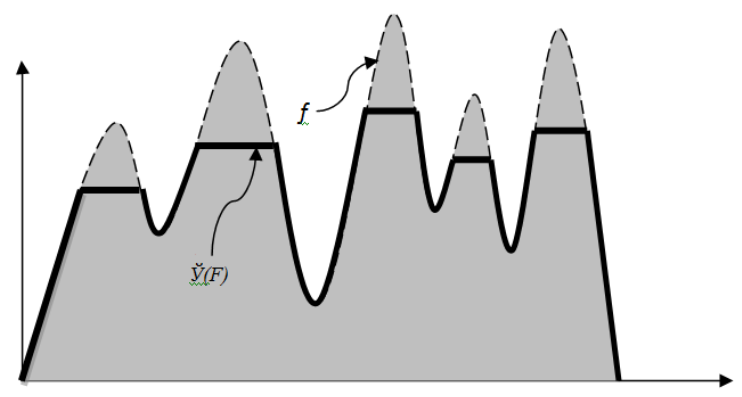

Fig. 3.1 Opening by reconstruction

\section{Structuring Elements:}

The structuring element is the basic neighborhood structure associated with morphological image operation. It is usually represented as a small matrix, whose shape and size impact the result of applying a certain morphological operator to an image. An essential part of the dilation and erosion operations is the structuring element used to probe the input image. A structuring element is a matrix consisting of only 0 's and 1 's that can have any arbitrary shape and size. The center pixel of the structuring element, called the origin, identifies the pixel of interest -- the pixel being processed. The pixels in the structuring element containing 1 's define the neighbourhood of the structuring element.

\section{FLOW CHART}

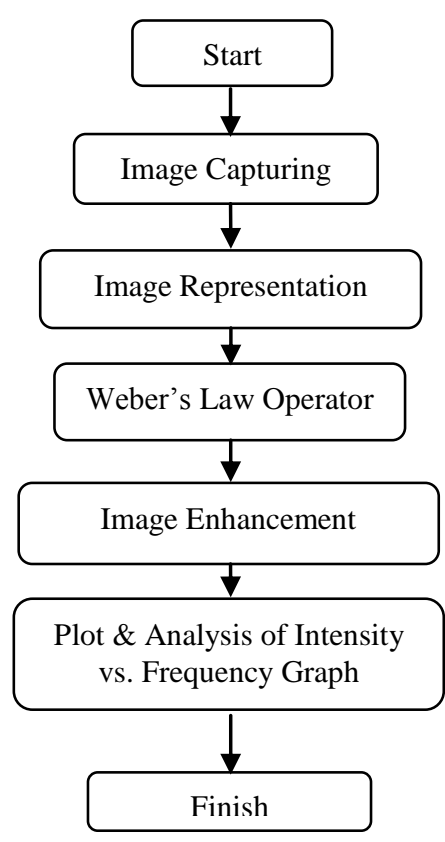

\section{PERFORMANCE ANALYSIS}

Performance of Enhanced Image is analyzed by Mean Square Error [MSE], Peak Signal to Noise Ratio [PSNR] along with the Processing time .i.e. Elapsed time for Execution

\section{CONCLUSION}

In this paper, various images with different background image with Low Intensity condition processed through Block Analysis method, Morphological Operation and Opening by Reconstruction. Finally Opening by reconstruction is used to analyze multi background definition of images. These enhanced images are also illustrated through filter.

\section{REFERENCES}

[1] Susanta Mukhopadhyay, Bhabatosh Chanda," A multiscale morphological approach to local contrast enhancement Signal Processing 80 (2000) 685 6 696"

[2] Angélica R. Jiménez-Sánchez, Jorge D. Mendiola-Santibañez, Iván R. Terol- Villalobos, Gilberto Herrera-Ruíz, Damián VargasVázquez, Juan J. García-Escalante, and Alberto Lara-Guevara, "Morphological Background Detection and enhancement of Images with Poor Lighting” IEEE Trans. Image Process. 18(3), pp. 613-623 (2009)

[3] Divya Madan,x` Susheel Kumar,“A New Approach for Improvement of Dark Images" , IJEEMF, Vol. 02, Issue 01, July 2012 ISSN: 2278-3989.

[4] K.Narasimhan, C.R.Sudarsan, Nagarajan Rajull "A Comparision of contrst Enhancement Techniques in poor illuminated gray level and color images"”, in IJCA, vol.24.no.2, 2011.

[5] K.Sreedhar and B.Panlal, Enhancement of images using morphological transformationl .IJCS\&IT, vol. 4, no. 1, 2012.

[6] Priyanka* Manoj Arora** "A MORPHOLOGICAL OPERATOR BASED APPROACH TO DETECT BACKGROUND FROM DARKEN IMAGES" Vol. 1 | No. 1 | July 2012 ISSN: 2278-6244

[7] Vaishnavi* Dr. P. Eswaran "Improved Colour Image Enhancement Scheme using Mathematical Morphology ", Volume 3, Issue 4, April 2013 IJARCSSE.

[8] V. Karthikeyan*1,V.J.Vijayalakshmi*2, P.Jeyakumar*3 A Novel Approach For The Enrichment Of Digital Images Using Morphological Operators International Journal of Engineering Trends and Technology- Volume4Issue3- 2013 ISSN: 2231-5381

[9] Dharamvir, Morphological Detection in Images International Conference on Current Trends in Advanced Computing "ICCTAC2013" 